energy scattering requires these trajectories not to be associated with spin-zero particles. ${ }^{21}$ This is by no means a drawback to our model; again, as in the case of the $\omega$

${ }^{21}$ R. J. N. Phillips and W. Rarita, Phys. Rev. 139, B1336 (1965). meson, we remark that we do not expect to obtain all the known mesons out of the present model. The dynamical origin of spin-zero particles may very well be found in a different channel.

\title{
Spontaneous Symmetry Breakdown without Massless Bosons*
}

\author{
Peter W. Higgs $\dagger$ \\ Department of Physics, University of North Carolina, Chapel Hill, North Carolina
}

(Received 27 December 1965)

\begin{abstract}
We examine a simple relativistic theory of two scalar fields, first discussed by Goldstone, in which as a result of spontaneous breakdown of $U(1)$ symmetry one of the scalar bosons is massless, in conformity with the Goldstone theorem. When the symmetry group of the Lagrangian is extended from global to local $U(1)$ transformations by the introduction of coupling with a vector gauge field, the Goldstone boson becomes the longitudinal state of a massive vector boson whose transverse states are the quanta of the transverse gauge field. A perturbative treatment of the model is developed in which the major features of these phenomena are present in zero order. Transition amplitudes for decay and scattering processes are evaluated in lowest order, and it is shown that they may be obtained more directly from an equivalent Lagrangian in which the original symmetry is no longer manifest. When the system is coupled to other systems in a $U(1)$ invariant Lagrangian, the other systems display an induced symmetry breakdown, associated with a partially conserved current which interacts with itself via the massive vector boson.
\end{abstract}

\section{INTRODUCTION}

$T^{1}$ HE idea that the apparently approximate nature of the internal symmetries of elementary-particle physics is the result of asymmetries in the stable solutions of exactly symmetric dynamical equations, rather than an indication of asymmetry in the dynamical equations themselves, is an attractive one. Within the framework of quantum field theory such a "spontaneous" breakdown of symmetry occurs if a Lagrangian, fully invariant under the internal symmetry group, has such a structure that the physical vacuum is a member of a set of (physically equivalent) states which transform according to a nontrivial representation of the group. This degeneracy of the vacuum permits nontrivial multiplets of scalar fields (which may be either fundamental dynamic variables or polynomials constructed from them) to have nonzero vacuum expectation values, whose appearance in Feynman diagrams leads to symmetry-breaking terms in propagators and vertices. That vacuum expectation values of scalar fields, or "vacuons," might play such a role in the breaking of symmetries was first noted by Schwinger ${ }^{1}$ and by Salam and Ward. ${ }^{2}$ Under the alternative name, "tadpole" diagrams, the graphs in which vacuons

* This work was partially supported by the U. S. Air Force Office of Scientific Research under grant No. AF-AFOSR-153-64. $\dagger$ On leave from the Tait Institute of Mathematical Physics, University of Edinburgh, Scotland.

${ }_{1}$ J. Schwinger, Phys. Rev. 104, 1164 (1954); Ann. Phys. (N. Y.) 2, 407 (1957).

2 A. Salam and J. C. Ward, Phys. Rev. Letters 5, 390 (1960); Nuovo Cimento 19, 167 (1961). appear have been used by Coleman and Glashow to account for the observed pattern of deviations from $S U(3)$ symmetry.

The study of field theoretical models which display spontaneous breakdown of symmetry under an internal Lie group was initiated by Nambu, ${ }^{4}$ who had noticed ${ }^{5}$ that the BCS theory of superconductivity ${ }^{6}$ is of this type, and was continued by Glashow ${ }^{7}$ and others. ${ }^{8}$ All these authors encountered the difficulty that their theories predicted, inter alia, the existence of a number of massless scalar or pseudoscalar bosons, named "zerons" by Freund and Nambu.9 Since the models which they discussed, being inspired by the BCS theory, used an attractive interaction between massless fermions and antifermions as the mechanism of symmetry breakdown, it was at first unclear whether zerons occurred as a result of the approximations (including the usual cutoff for divergent integrals) involved in handling the models or whether they would still be there in an exact solution. Some authors,

${ }^{3}$ S. Coleman and S. L. Glashow, Phys. Rev. 134, B671 (1964). ${ }^{4} \mathrm{Y}$. Nambu and G. Jona-Lasinio, Phys. Rev. 122, 345 (1961); 124, 246 (1961); Y. Nambu and P. Pascual, Nuovo Cimento 30, $354(1963)$.

5 Y. Nambu, Phys. Rev. 117, 648 (1960).

${ }^{6}$ J. Bardeen, L. N. Cooper, and J. R. Schrieffer, Phys. Rev. $106,162(1957)$.

${ }^{7} \mathrm{M}$. Baker and S. L. Glashow, Phys. Rev. 128, 2462 (1962); S. L. Glashow, ibid. 130, 2132 (1962).

${ }_{8}^{8}$ M. Suzuki, Progr. Theoret. Phys. (Kyoto) 30, 138 (1963); 30, 627 (1963); N. Byrne, C. Iddings, and E. Shrauner, Phys. Rev. 139, B918 (1965); 139, B933 (1965).

9 P. G. O. Freund and Y. Nambu, Phys. Rev. Letters 13, 221 (1964). 
wishing to identify their zerons with known massive scalar or pseudoscalar mesons, were prepared to spoil the elegance of their theories by adding symmetrybreaking terms to the Lagrangian in order to generate masses.

That zerons must indeed be present in Lorentz invariant field theories in which an internal symmetry breaks down spontaneously was first shown by Goldstone..$^{10} \mathrm{He}$ clarified the nature of the phenomenon considerably by exhibiting it in a model of a selfinteracting scalar field, where the vacuon is the vacuum expectation value of the field itself, rather than that of a bilinear combination of fermion operators which occurs in the BCS model and its progeny. In a theory of this type the breakdown of symmetry occurs already at the level of classical field theory, where vacuons are just nontrivial translationally invariant solutions of the field equations, and zerons, whose existence is readily demonstrated, are small-amplitude waves (superimposed on a "vacuon" solution) whose frequency tends to zero as their wavelength tends to infinity. In a later paper ${ }^{11}$ the proof of the Goldstone theorem, as it is now known, was generalized to allow for the possibility that vacuons might be formed from polynomials of any degree in the fundamental field variables of a dynamical system.

During the last few years the problem of how to avoid massless Goldstone bosons has received much attention. Attempts in this direction have been encouraged by the observation that the BCS model does not contain such excitations, provided that Coulomb interactions are taken into account. ${ }^{12}$ Klein and Lee ${ }^{13}$ showed that in a nonrelativistic theory the spectral representations upon which the more sophisticated proofs of Goldstone's theorem are based are not so restricted in form as to allow the proof to go through, and they conjectured that this might remain true in some relativistic theories. But Gilbert ${ }^{14}$ pointed out that their extra terms are ruled out in relativistic theories by the requirement of manifest Lorentz covariance. The present writer restored the status quo to a limited extent by remarking ${ }^{15}$ that radiation gauge formulations of gauge field theories, of which electrodynamics is the simplest and best known example, can describe Lorentz-invariant dynamical systems despite the lack of manifest covariance of some of the equations. The freedom which Klein and Lee hoped for in the spectral representations is thereby restored sufficiently to invalidate the Goldstone theorem. From a more physical standpoint one may regard this as an effect of Coulomb interactions, treated now as part of a relativistic field theory.

${ }^{10} \mathrm{~J}$. Goldstone, Nuovo Cimento 19, 154 (1961).

$1 \mathrm{~J}$. Goldstone, A. Salam, and S. Weinberg, Phys. Rev. 127, 9.65 (1962).

12 P. W. Anderson, Phys. Rev. 112, 1900 (1958).

13 A. Klein and B. W. Lee, Phys. Rev. Letters 12, 266 (1964).

14 W. Gilbert, Phys. Rev. Letters 12, 713 (1964).

${ }_{15}$ P. W. Higgs, Phys. Letters 12, 132 (1964).
More recently Guralnik, Hagen, and Kibble ${ }^{16}$ and Lange $^{17}$ have studied how the failure of global (as distinct from local) conservation laws in spontaneous breakdown theories is related to the existence of Goldstone bosons. Meanwhile, proofs of the theorem have reached new levels of sophistication within the language (or languages) of axiomatic field theory. ${ }^{18} \mathrm{It}$ has been pointed out that theories of the type proposed in Ref. 15 do not contradict the Goldstone theorem, but rather represent a departure from the assumptions, such as manifest covariance and manifest causality, upon which it is based. Such considerations do not seem relevant to the possible usefulness of such theories in generating zeron-free models of spontaneous symmetry breakdown, a point which seems to have been overlooked by those ${ }^{19}$ who proclaim the failure of the Nambu-Glashow program.

In parallel with the development of "superconductor" models a program has emerged for describing the weak, ${ }^{20}$ and possibly also the strong, ${ }^{21}$ interactions by an extension of the gauge principle ${ }^{22}$ which operates in electrodynamics. According to this principle the symmetry group of the Lagrangian is to be enlarged from global to local (i.e., coordinate-dependent) transformations: To maintain the invariance of derivative terms it is necessary to couple the currents of the group generators to a multiplet of vector fields belonging to the adjoint representation. ${ }^{23}$ Like the "superconductor" theories, these gauge theories have suffered from a zero-mass difficulty: The gauge principle appears to guarantee that the associated vector field quanta are massless, in

${ }^{16}$ G. S. Guralnik, C. R. Hagen, and T. W. B. Kibble, Phys. Rev. Letters 13, 585 (1964). These authors appear to attribute the failure of a local conservation law to yield a global conservation law to the lack of manifest covariance of a theory. In fact this happens even in manifestly covariant models of spontaneous breakdown.

${ }^{17}$ R. V. Lange, Phys. Rev. Letters 14, 3 (1965).

${ }^{18}$ R. F. Streater, Proc. Roy. Soc. (London) A287, 510 (1965). The proof of the Goldstone theorem given here is based on axioms which include manifest causality. Radiation gauge theories escape this version of the theorem by virtue of their (quite innocuous) acausality. The question of the extent to which one may give up manifest covariance and causality in a theory without losing covariance and causality of the physics which it describes deserves further study. If there are contexts in which it is possible other than the gauge theories which we are discussing, then there are probably other escape routes from the Goldstone theorem. See also D. Kastler, D. W. Robinson, and A. Swieca, Commun. Math. Phys. (to be published).

${ }^{19}$ R. F. Streater, Phys. Rev. Letters 15, 475 (1965). The generalized Goldstone theorem proved by this author and extended by N. Fuchs, Phys. Rev. Letters 15, 911 (1965) also relies on manifest causality and is therefore inapplicable to gauge theories.

${ }^{20}$ S. A. Bludman, Phys. Rev. 100, 372 (1955); Nuovo Cimento 9, 433 (1958); S. L. Glashow, Nucl. Phys. 10, 107 (1959); 22, 579 (1961).

${ }^{21}$ A. Salam and J. C. Ward, Nuovo Cimento 11, 568 (1959); Phys. Rev. 136, B'763 (1964); J. J. Sakurai, Ann. Phys. (N. Y.) 11, 1 (1960).

${ }^{22}$ C. N. Yang and R. L. Mills, Phys. Rev. 96, 191 (1954); R. Shaw, dissertation, Cambridge University, 1954 (unpublished); R. Utiyama, Phys. Rev. 101, 1597 (1956).

${ }^{23}$ M. Gell-Mann and S. L. Glashow, Ann. Phys. (N. Y.) 15, 437 (1961). 
perturbation theory at least. But the only known massless vector boson is the photon; the existing evidence suggests ${ }^{24}$ that all other vector bosons must be massive. In particular, the hypothetical intermediate vector bosons of weak interactions, which in a gauge theory belong to the gauge field multiplet, must be much heavier than the known hadrons. For the most part, advocates of gauge theories have met this difficulty either by spoiling the gauge invariance of their theories with explicit mass terms or by taking comfort from the argument of Schwinger ${ }^{25}$ that a sufficiently strong gauge-field coupling might generate mass. Recently, however, it was shown by Englert and Brout ${ }^{26}$ and by the present writer ${ }^{27}$ that gauge vector mesons acquire mass if the symmetry to whose generators they are coupled breaks down spontaneously, however weak their coupling may be. In Ref. 27 this phenomenon was exhibited in a classical field theory, and it was pointed out that in such a theory the longitudinal polarization of the massive vector excitation replaces the massless scalar excitation which would occur in the absence of coupling to the gauge field. Thus it now appears that the spontaneous breakdown program of Nambu et al. and the gauge field program of Salam et al. stand or fall together. Each saves the other from its zero-mass difficulty.

The purpose of the present paper is to amplify and substantiate the assertions made in Refs. 15 and 27 by displaying the behavior of the simplest possible relativistic field theory which combines spontaneous breakdown of symmetry under a compact Lie group with the gauge principle. That is to say, we take the symmetry group to be the trivial Abelian group $U(1)$, we take as the fundamental dynamic variables a pair of Hermitian scalar fields $\Phi_{1}(x), \Phi_{2}(x)$ together with the Hermitian vector gauge field $A_{\mu}(x)$, and we induce spontaneous breakdown by means of the simplest $U(1)$ invariant self-interaction of $\Phi_{a}(x)$ which will do the trick, namely, a combination of quadratic and quartic terms. In the absence of the gauge field coupling, the model is just one which Goldstone ${ }^{10}$ first discussed. ${ }^{28}$

In Sec. II the behavior of the small-amplitude wave solutions of the classical field equations is used as a guide in formulating a perturbation theory in which the major effects of spontaneous breakdown are already taken into account in zero order. The radiation gauge commutators of the zero-order approximation are obtained and used to provide an explicit realization of the spectral representation which was proposed in Ref. 15. In Sec. III decay and scattering amplitudes are calculated in lowest order and it is verified that they are

\footnotetext{
${ }^{24}$ See, for example, S. Weinberg, Phys. Rev. Letters 13, 495 (1964).

25 J. Schwinger, Phys. Rev. 125, 397 (1962); 128, 2425 (1962).

${ }_{26}$ F. Englert and R. Brout, Phys. Rev. Letters 13, 321 (1964).

27 P. W. Higgs, Phys. Rev. Letters 13, 508 (1964).

28 I understand from Dr. Goldstone (private communication) that he and W. Gilbert at one time considered adding a gauge field to the model.
}

gauge-invariant, Lorentz-invariant, and causal despite the lack of manifest covariance and causality of the radiation gauge. In Sec. IV it is shown that the same amplitudes may be derived by a manifestly covariant and causal method from an equivalent Lagrangian which lacks the original symmetry. Finally, our conclusions are summarized in Sec. V, and the way in which coupling between a system of the kind described here and other symmetric dynamical systems may lead to partially conserved currents is sketched.

In subsequent papers we propose to elaborate these considerations, both with regard to symmetry groups and with regard to mechanisms of symmetry breakdown, so as to make closer contact with particle physics.

\section{THE MODEL}

The Lagrangian density from which we shall work is given $\mathrm{by}^{29}$

$$
\begin{aligned}
\mathscr{L}=-\frac{1}{4} g^{\kappa \mu} g^{\lambda \nu} F_{\kappa \lambda} F_{\mu \nu}-\frac{1}{2} g^{\mu \nu} \nabla_{\mu} \Phi_{a} \nabla_{\nu} \Phi_{a} & \\
& +\frac{1}{4} m_{0}^{2} \Phi_{a} \Phi_{a}-\frac{1}{8} f^{2}\left(\Phi_{a} \Phi_{a}\right)^{2} .
\end{aligned}
$$

In Eq. (1) the metric tensor $g^{\mu \nu}=-1(\mu=\nu=0)$, $+1(\mu=\nu \neq 0)$ or $0(\mu \neq \nu)$, Greek indices run from 0 to 3 and Latin indices from 1 to 2 . The $U(1)$-covariant derivatives $F_{\mu \nu}$ and $\nabla_{\mu} \Phi_{a}$ are given by

$$
\begin{aligned}
F_{\mu \nu} & =\partial_{\mu} A_{\nu}-\partial_{\nu} A_{\mu}, \\
\nabla_{\mu} \Phi_{1} & =\partial_{\mu} \Phi_{1}-e A_{\mu} \Phi_{2}, \\
\nabla_{\mu} \Phi_{2} & =\partial_{\mu} \Phi_{2}+e A_{\mu} \Phi_{1} .
\end{aligned}
$$

At first sight this theory appears to be scalar electrodynamics augmented by a quartic self-interaction. However, what appears to be the bare-mass term has the wrong sign. In conjunction with the quartic term this feature has the consequence that the field equations

$$
\begin{gathered}
\partial_{\nu} F^{\mu \nu}=e j^{\mu}, \quad j_{\mu}=\Phi_{2} \nabla_{\mu} \Phi_{1}-\Phi_{1} \nabla_{\mu} \Phi_{2}, \\
\nabla_{\mu} \nabla^{\mu} \Phi_{a}+\frac{1}{2}\left(m_{0}^{2}-f^{2} \Phi_{b} \Phi_{b}\right) \Phi_{a}=0,
\end{gathered}
$$

in the classical theory possess a coordinate-independent solution

$$
A_{\mu}=0, \quad \Phi_{b} \Phi_{b}=m_{0}^{2} / f^{2} .
$$

The invariance of the Lagrangian (1) under the local $U(1)$ transformations

$$
\begin{aligned}
& A_{\mu}(x) \rightarrow A_{\mu}(x)+e^{-1} \partial_{\mu} \Lambda(x), \\
& \Phi_{1}(x) \rightarrow \Phi_{1}(x) \cos \Lambda(x)+\Phi_{2}(x) \sin \Lambda(x), \\
& \Phi_{2}(x) \rightarrow-\Phi_{1}(x) \sin \Lambda(x)+\Phi_{2}(x) \cos \Lambda(x),
\end{aligned}
$$

is reflected in the existence of a one-parameter family of static solutions defined by Eq. (3). ${ }^{30}$ In the classical

${ }^{29}$ We do not explicitly perform the symmetrizations which a correct quantum-mechanical treatment would demand. They are not necessary for the purposes of the present paper and would, in any case, be dealt with more satisfactorily in a first-order formalism.

${ }^{30}$ Strictly speaking, global $U(1)$ invariance suffices to guarantee this result. 
theory, any one of these solutions,

$$
\Phi_{1}=\eta \cos \alpha, \quad \Phi_{2}=\eta \sin \alpha,
$$

where $\eta=m_{0} / f$, defines a possible asymmetric configuration of stable equilibrium, stability being ensured by the sign of the quartic term in Eq. (1). Quantum mechanically each solution, regarded as the "bare" value of the vacuon $\left\langle\Phi_{a}\right\rangle$, corresponds to a different possible vacuum state. ${ }^{31}$

Let us choose $\alpha=\pi / 2$ and linearize the classical field equations (2) by treating $A_{\mu}, \Phi_{1}$, and $\Phi_{2}-\eta$ as small quantities. We obtain

$$
\begin{gathered}
\partial_{\nu} F^{\mu \nu}=-e^{2} \eta^{2} B^{\mu}, \quad \partial_{\mu} B^{\mu}=0, \\
\left(\square-m_{0}^{2}\right) \chi=0,
\end{gathered}
$$

in which we have introduced the notation

$$
\begin{aligned}
B_{\mu} & =A_{\mu}-(e \eta)^{-1} \partial_{\mu} \Phi, \\
\Phi & =\Phi_{1}, \quad \chi=\Phi_{2}-\eta .
\end{aligned}
$$

As we remarked in Ref. 27, these are the linear field equations which, after quantization, describe free vector bosons of mass $e \eta$ and free scalar bosons of mass $m_{0}$. The longitudinal vector excitation becomes the Goldstone scalar excitation in the limit $e \rightarrow 0$. The Lagrangian to which these field equations belong is given by

$$
\begin{array}{r}
\mathfrak{L}_{0}=-\frac{1}{4} F_{\mu \nu} F^{\mu \nu}-\frac{1}{2} g^{\mu \nu}\left(\partial_{\mu} \Phi-m_{1} A_{\mu}\right)\left(\partial_{\nu} \Phi-m_{1} A_{\nu}\right) \\
-\frac{1}{2} g^{\mu \nu} \partial_{\mu} \chi \partial_{\nu} \chi-\frac{1}{2} m_{0}{ }^{2} \chi^{2},
\end{array}
$$

where we have written $m_{1}$ for the vector boson mass $e \eta$.

The foregoing analysis of classical small-amplitude wave propagation suggests the following perturbation theoretic treatment of the quantized theory. We rewrite Eq. (1) in the form $\mathfrak{L}=\mathfrak{L}_{0}+\mathfrak{L}_{\text {int }}$, where $\mathfrak{L}_{0}$ is given by Eq. (6) apart from a trivial additive constant and

$$
\begin{gathered}
\mathscr{L}_{\mathrm{int}}=e A^{\mu}\left(\chi \partial_{\mu} \Phi-\Phi \partial_{\mu} \chi\right)-e m_{1} \chi A_{\mu} A^{\mu}-\frac{1}{2} f m_{0} \chi\left(\Phi^{2}+\chi^{2}\right) \\
-\frac{1}{2} e^{2} A_{\mu} A^{\mu}\left(\Phi^{2}+\chi^{2}\right)-\frac{1}{8} f^{2}\left(\Phi^{2}+\chi^{2}\right)^{2} .
\end{gathered}
$$

Note that the ancestry of (6) plus (7) in the symmetric Lagrangian (1) is embodied in the relation $m_{1} / m_{0}=e / f$ between the bare masses and the bare coupling constants. Our perturbation theory now consists in developing transition amplitudes in powers of $e$ and $f$

31 The orthogonality of the worlds built upon different vacua may be understood as a consequence of the impossibility in the classical theory of a displacement of the system from one static configuration to another, on account of the infinite inertia associated with such a motion. To see this, imagine a one-dimensional model consisting of an infinite uniform elastic string subjected to a force field of cylindrical symmetry about the axis from which transverse displacements $\Phi_{1}, \Phi_{2}$ are measured. (We omit the gauge field from this do-it-yourself model.) If the force is such that stable equilibrium occurs when the whole string is at a distance $\eta$ from the axis in any direction, then the system exhibits broken rotational symmetry. Displacement of the string from equilibrium at one orientation to equilibrium at another is impossible, since the moment of inertia about the axis is infinite. Waves on the string do not conserve angular momentum about the axis, since the string as a whole can emit or absorb angular momentum without recoiling. (or, equivalently, in inverse powers of $\eta$ ), the masses $m_{0}$ and $m_{1}$ being treated as of order zero. ${ }^{32}$ Thus in Eq. (7) all five cubic vertices are of the first degree and all five quartic vertices are of the second in the expansion parameter. It will be found that, with few exceptions, gauge-invariant results are obtained only when all Feynman graphs of the same degree are summed.

We first write down the commutators and propagators corresponding to the bare Lagrangian $\mathfrak{L}_{0}$. Apart from the terms in $\chi$, this is just the second-order Lagrangian of a model proposed by Boulware and Gilbert $^{33}$ as an illustration of the possibility of a gaugeinvariant theory describing a massive vector boson. We shall study it in a radiation gauge; the Lorentz gauge formulation, which even in quantum electrodynamics leads to unnecessary complications such as redundant states, is here inconsistent with the canonical com, mutation rules, as was pointed out by Guralnik, Hagenand Kibble. ${ }^{16}$ In a radiation gauge defined by the condition

$$
(\partial A)+(n \partial)(n A)=0,
$$

where $n^{\mu}$ is a constant timelike unit vector and $(a b)$ denotes $a_{\mu} b^{\mu}$, the variables $A_{\mu}$ and $\Phi$ may be expressed in terms of the massive vector field $B_{\mu}$ which was introduced in Eq. (5):

$$
\begin{gathered}
A_{\mu}=B_{\mu}+m_{1}^{-1} \partial_{\mu} \Phi, \\
\Phi=-m_{1}\left[\left(\partial^{2}\right)+(n \partial)^{2}\right]^{-1}[(\partial B)+(n \partial)(n B)] .
\end{gathered}
$$

Since $\mathfrak{L}_{0}$, when expressed in terms of $B_{\mu}$ and $\chi$, is just the usual second order Lagrangian for free vector and scalar bosons, we may immediately write down the covariant commutators:

$$
\begin{aligned}
{\left[B_{\mu}(x), B_{\nu}(y)\right] } & =-i\left(g_{\mu \nu}-m_{1}^{-2} \partial_{\mu} \partial_{\nu}\right) \Delta\left(x-y, m_{1}^{2}\right), \\
{[\chi(x), \chi(y)] } & =-i \Delta\left(x-y, m_{0}^{2}\right),
\end{aligned}
$$

where $\Delta\left(x, m^{2}\right)=i(2 \pi)^{-3} \int d^{4} k e^{i(k x)} \epsilon\left(k^{0}\right) \delta\left(k^{2}+m^{2}\right)$. Then Eq. (8) enables us to deduce the nonvanishing commutators of $A_{\mu}, \Phi$, and $\chi$ :

$$
\begin{aligned}
{\left[A_{\mu}(x), A_{\nu}(y)\right]=} & -i\left\{g_{\mu \nu}-\left[\left(n_{\mu} \partial_{\nu}+n_{\nu} \partial_{\mu}\right)(n \partial)+\partial_{\mu} \partial_{\nu}\right]\right. \\
& \left.\times\left[\left(\partial^{2}\right)+(n \partial)^{2}\right]^{-1}\right\} \Delta\left(x-y, m_{1}^{2}\right) \\
{\left[A_{\mu}(x), \Phi(y)\right]=} & -i m_{1} n_{\mu}(n \partial) \\
& \times\left[\left(\partial^{2}\right)+(n \partial)^{2}\right]^{-1} \Delta\left(x-y, m_{1}^{2}\right), \\
{[\Phi(x), \Phi(y)]=} & -i(n \partial)^{2}\left[\left(\partial^{2}\right)+(n \partial)^{2}\right]^{-1} \Delta\left(x-y, m_{1}^{2}\right), \\
{[\chi(x), \chi(y)]=} & -i \Delta\left(x-y, m_{0}^{2}\right) .
\end{aligned}
$$

We also note the commutator relation

$$
\begin{aligned}
{\left[B_{\mu}(x), \Phi(y)\right]=- } & -i m_{1}^{-1}\left[\left(\partial^{2}\right) n_{\mu}-(n \partial) \partial_{\mu}\right](n \partial) \\
& \times\left[\left(\partial^{2}\right)+(n \partial)^{2}\right]^{-1} \Delta\left(x-y, m_{1}^{2}\right) .
\end{aligned}
$$

${ }^{32}$ When one comes to consider radiative corrections, it becomes necessary to make these statements about the renormalized rather than the bare masses and coupling constants.

${ }^{33}$ D. C. Boulware and W. Gilbert, Phys. Rev. 126, 1563 (1962). 
The generator $Q(t)$ of infinitesimal global $U(1)$ transformations (that is, transformations (4) with $\Lambda$ constant and infinitesimal) on the hypersurface $(n x)+t=0$ is $\int d \sigma_{\mu} j^{\mu}$, where $d \sigma_{\mu}$ is the volume element of the hypersurface and $j_{\mu}(x)$ is given by Eq. (2). The invariance of the Lagrangian (1) under these transformations leads to the local conservation law, $\partial_{\mu} j^{\mu}=0$. However, even in the absence of the gauge field coupling, the four-dimensional integral of this equation fails to yield the usual global conservation law, $Q(t)$ $=$ constant, since the flux of $j_{\mu}$ across the surface of a large sphere bounding the hypersurface does not tend to zero as the radius tends to infinity. That this is so can be seen by noting that the lowest order approximation to $j_{\mu}$ is $-e \eta^{2} B_{\mu}$ (or $\eta \partial_{\mu} \Phi$ in the absence of the gauge field): Matrix elements of this operator do not decrease sufficiently rapidly at large spatial distances for the flux term to vanish. (In normal theories the lowest order term in $j_{\mu}$ is quadratic, giving a better asymptotic behavior of the matrix elements.) Strictly speaking, the "operator" $Q(t)$ is now not merely timedependent but nonexistent, since $\int d \sigma_{\mu} j^{\mu}$ diverges as a result of the same bad asymptotic behavior of $j^{\mu}$. However, certain commutators, such as $\left[Q(t), \Phi_{a}(y)\right]$, do still exist. ${ }^{34}$

The zero-order approximation to the commutator vacuum expectation value $\left\langle i\left[j_{\mu}(x), \Phi_{1}(y)\right]\right\rangle$, upon which so much of the discussion of the Goldstone theorem has centered, ${ }^{35}$ is found by replacing $j_{\mu}$ by $-e \eta^{2} B_{\mu}$ and using Eq. (11): It is

$$
\eta\left[(n \partial) \partial_{\mu}-\left(\partial^{2}\right) n_{\mu}\right](n \partial)\left[\left(\partial^{2}\right)+(n \partial)^{2}\right]^{-1} \Delta\left(x-y, m_{1}^{2}\right) .
$$

Its Fourier transform,

$$
\begin{aligned}
-2 \pi \eta\left[(n k) k_{\mu}-\right. & \left.\left(k^{2}\right) n_{\mu}\right](n k) \\
& \times\left[\left(k^{2}\right)+(n k)^{2}\right]^{-1} \epsilon\left(k^{0}\right) \delta\left(k^{2}+m_{1}^{2}\right),
\end{aligned}
$$

provides an explicit realization of a spectral representation of the form obtained in Ref. 15, the Lorentz invariance of the spectrum of intermediate states now being made clear. We are led to conjecture that the vacuum expectation value of the exact commutator may be of the form

$$
\begin{aligned}
\left\langle\Phi_{2}\right\rangle\left[(n \partial) \partial_{\mu}-\left(\partial^{2}\right) n_{\mu}\right](n \partial)\left[\left(\partial^{2}\right)+(n \partial)^{2}\right]^{-1} & \\
& \times \int_{0}^{\infty} d m^{2} \rho\left(m^{2}\right) \Delta\left(x-y, m^{2}\right),
\end{aligned}
$$

where $\rho\left(m^{2}\right)$ is a nonnegative spectral function satisfy-

${ }^{34}$ In Ref. 18 it is proved that in a manifestly causal theory this commutator (or at least certain of its matrix elements) is independent of $t$, despite the breakdown of the global conservation law. The gauge field coupling destroys manifest causality and induces a time dependence in this commutator: In the zeroorder approximation it oscillates at a frequency $m_{1}$.

${ }^{35}$ See Refs. 11, 13, 14, and 15. In Refs. 14 and 15 it is implied erroneously that the commutator $\left[Q(t), \Phi_{a}(y)\right]$ is independent of $t$. Fortunately, the discussion of the Goldstone theorem in these papers does not depend on this assumption. ing the sum rule

$$
\int_{0}^{\infty} d m^{2} \rho\left(m^{2}\right)=1
$$

It may be noted that when $m_{1}=0$ in Eq. (12), corresponding to $e=0$, we recover the manifestly covariant spectral representation $-2 \pi \eta k_{\mu} \epsilon\left(k^{0}\right) \delta\left(k^{2}\right)$ and with it the Goldstone theorem.

We define the propagators of the system described by $\mathscr{L}_{0}$ to be the quantities $\left\langle T^{*} A_{\mu}(x) A_{\nu}(y)\right\rangle$, etc., obtained from the corresponding commutators in Eq. (10) by substituting for $\Delta$ the scalar propagator $\Delta_{F}$ given by

$$
\Delta_{F}\left(x, m^{2}\right)=(2 \pi)^{-4} \int d^{4} k e^{i(k x)}\left(k^{2}+m^{2}-i \epsilon\right)^{-1} .
$$

Then we may calculate $S$-matrix elements by using the simple Feynman rules based on the Nishijima-Wick expansion of the expression $T^{*} \exp \left(i \int d^{4} x \mathfrak{L}_{\mathrm{int}}\right)$ for the $S$-operator in the interaction picture. ${ }^{36}$ We thereby avoid the $n_{\mu}$-dependent terms, in addition to those already introduced by the radiation gauge, which the use of simple chronological ordering and the DysonWick expansion would entail.

\section{DECAY AND SCATTERING AMPLITUDES}

As an illustration of the physical content of the model we now calculate in lowest order the matrix elements for the simplest processes which it describes. We shall verify that, despite the unpromising appearance of the radiation gauge propagators, these matrix elements are gauge invariant and Lorentz invariant.

In applying the Feynman rules we shall need, in addition to the propagators, the wave functions $a_{\mu}(k, \sigma)$ and $\phi(k, \sigma)$ which correspond to the annihilation by the operators $A_{\mu}$ and $\Phi$, respectively, of a vector meson from an incoming state of momentum $k$ and spin component $\sigma$. They are related by the Fourier transform of Eq. (8) to the usual vector meson wave function $b_{\mu}(k, \sigma)$, which has the explicit form

$$
\begin{aligned}
b^{\mu}(k, 0) & =\left(\omega / m_{1}\right)(|\mathbf{k}| / \omega, \mathbf{k} /|\mathbf{k}|), \\
b^{\mu}(k, \pm 1) & =2^{-1 / 2}\left(0, \mathbf{e}_{1} \pm i \mathbf{e}_{2}\right),
\end{aligned}
$$

where $\omega=\left(|\mathbf{k}|^{2}+m_{1}^{2}\right)^{1 / 2}$ and $\mathbf{e}_{1}, \mathbf{e}_{2}, \mathbf{k} /|\mathbf{k}|$ form a righthanded orthonormal triad. Actually, all that we shall need is the relation

$$
a_{\mu}=b_{\mu}+\left(i k_{\mu} / m_{1}\right) \phi
$$

by which matrix elements may be expressed in terms of wave functions $b_{\mu}$ and $\phi$, the desired invariance being achieved by the cancellation of all terms containing factors $\phi$. Similar considerations apply to out-

${ }^{36}$ P. T. Matthews, Phys. Rev. 76, 684 (1949); K. Nishijima Progr. Theoret. Phys. (Kyoto) 5, 405 (1950). The most general conditions for the validity of this expression have been stated by C. S. Lam, Nuovo Cimento 38, 1755 (1965). 
going states and associated complex conjugate wave functions.

\section{i. Decay of a Scalar Boson into Two Vector Bosons}

The process occurs in first order (four of the five cubic vertices contribute), provided that $m_{0}>2 m_{1}$. Let $p$ be the incoming and $k_{1}, k_{2}$ the outgoing momenta. Then

$$
\begin{gathered}
M=i\left\{e\left[a^{* \mu}\left(k_{1}\right)\left(-i k_{2 \mu}\right) \phi^{*}\left(k_{2}\right)+a^{* \mu}\left(k_{2}\right)\left(-i k_{1 \mu}\right) \phi^{*}\left(k_{1}\right)\right]\right. \\
-e\left(i p_{\mu}\right)\left[a^{* \mu}\left(k_{1}\right) \phi^{*}\left(k_{2}\right)+a^{* \mu}\left(k_{2}\right) \phi^{*}\left(k_{1}\right)\right] \\
\left.-2 e m_{1} a_{\mu}^{*}\left(k_{1}\right) a^{* \mu}\left(k_{2}\right)-f m_{0} \phi^{*}\left(k_{1}\right) \phi^{*}\left(k_{2}\right)\right\} .
\end{gathered}
$$

By using Eq. (15), conservation of momentum, and the transversality $\left(k_{\mu} b^{\mu}(k)=0\right)$ of the vector wave functions we reduce this to the form

$$
\begin{aligned}
M=-2 i e m_{1} b^{* \mu}\left(k_{1}\right) b_{\mu} * & \left(k_{2}\right) \\
& -i e m_{1}^{-1}\left(p^{2}+m_{0}^{2}\right) \phi^{*}\left(k_{1}\right) \phi^{*}\left(k_{2}\right) .
\end{aligned}
$$

We have retained the last term, which we shall need in calculating scattering amplitudes; when the incident particle is on the mass shell it vanishes and we are left with the invariant expression

$$
M=-2 i e m_{1} b^{* \mu}\left(k_{1}\right) b_{\mu}{ }^{*}\left(k_{2}\right) .
$$

Conservation of angular momentum allows three possibilities for the spin states of the decay products: They may be both right-handed, both left-handed, or both longitudinal $\left(\sigma_{1}=\sigma_{2}=+1,-1\right.$, or 0$)$. With the help of the explicit vectors (14), we find

$$
\begin{gathered}
M(+1,+1)=M(-1,-1)=2 \text { iem }_{1}, \\
M(0,0)=i f m_{0}\left(1-2 e^{2} / f^{2}\right) .
\end{gathered}
$$

We note that as $e \rightarrow 0$ the amplitudes for decay to transverse states tend to zero, but the amplitude $M(0,0)$ tends to the value if $m_{0}$ which we would calculate from the vertex $-\frac{1}{2} f m_{0} \Phi^{2} \chi$ for the decay of one massive into two massless scalar bosons in the original Goldstone model. (The sign change arises from the factor $i$ which is associated with the term $\phi$ in each $b_{\mu}$.)

\section{ii. Vector Boson-Vector Boson Scattering}

Let $k_{1}, k_{2}$ be the incoming and $k_{1}{ }^{\prime}, k_{2}{ }^{\prime}$ the outgoing momenta. The process occurs as a second-order effect of the cubic vertices, by exchange of a scalar boson in the $s$, $t$, or $u$ channel, where $s=-\left(p_{1}+p_{2}\right)^{2}, t$ $=-\left(p_{1}-p_{1}\right)^{2}, u=-\left(p_{1}-p_{2}{ }^{\prime}\right)^{2}$. It also occurs as a direct effect of two of the quartic vertices. Equation (16) enables us to write down

$$
\begin{aligned}
M_{s}=i^{2}\left\{-2 e m_{1} b_{\mu}{ }^{*}\left(k_{1}{ }^{\prime}\right) b^{* \mu}\left(k_{2}{ }^{\prime}\right)\right. & \\
& \left.+e m_{1}^{-1}\left(s-m_{0}{ }^{2}\right) \phi^{*}\left(k_{1}{ }^{\prime}\right) \phi^{*}\left(k_{2}{ }^{\prime}\right)\right\} \\
& \times i\left(s-m_{0}\right)^{-1}\left\{-2 e m_{1} b_{\nu}\left(k_{1}\right) b^{\nu}\left(k_{2}\right)\right. \\
& \left.+e m_{1}{ }^{-1}\left(s-m_{0}{ }^{2}\right) \phi\left(k_{1}\right) \phi\left(k_{2}\right)\right\}
\end{aligned}
$$

and similar expressions for $M_{t}$ and $M_{u}$. The quartic vertices yield a contribution given by

$$
\begin{aligned}
M_{\text {direct }}= & i\left(-2 e^{2}\right)\left\{a_{\mu}{ }^{*}\left(k_{1}{ }^{\prime}\right) a^{* \mu}\left(k_{2}{ }^{\prime}\right) \phi\left(k_{1}\right) \phi\left(k_{2}\right)\right. \\
& +5 \text { similar terms }\} \\
& +i\left(-3 f^{2}\right) \phi^{*}\left(k_{1}{ }^{\prime}\right) \phi^{*}\left(k_{2}{ }^{\prime}\right) \phi\left(k_{1}\right) \phi\left(k_{2}\right) \\
=-2 i e^{2}\left\{b_{\mu}{ }^{*}\left(k_{1}{ }^{\prime}\right) b^{* \mu}\left(k_{2}{ }^{\prime}\right) \phi\left(k_{1}\right) \phi\left(k_{2}\right)\right. & \\
& +5 \text { similar terms }\} \\
& +i\left(4 e^{2}-3 f^{2}\right) \phi^{*}\left(k_{1}\right) \phi^{*}\left(k_{2}{ }^{\prime}\right) \phi\left(k_{1}\right) \phi\left(k_{2}\right) .
\end{aligned}
$$

It is only when we combine these four contributions that we obtain (after some algebra) the invariant expression

$$
\begin{aligned}
M_{\text {total }}= & M_{s}+M_{t}+M_{u}+M_{\text {direet }} \\
= & -4 i e^{2} m_{1}{ }^{2}\left\{\left(s-m_{0}{ }^{2}\right)^{-1} b^{* \mu}\left(k_{1}\right) b^{* \mu}\left(k_{2}{ }^{\prime}\right) b_{\nu}\left(k_{1}\right) b^{\nu}\left(k_{2}\right)\right. \\
& +\left(t-m_{0}\right)^{-1} b_{\mu}{ }^{*}\left(k_{1}{ }^{\prime}\right) b^{\mu}\left(k_{1}\right) b_{\nu}{ }^{*}\left(k_{2}{ }^{\prime}\right) b^{\nu}\left(k_{2}\right) \\
& \left.+\left(u-m_{0}^{2}\right)^{-1} b_{\mu}{ }^{*}\left(k_{1}^{\prime}\right) b^{\mu}\left(k_{2}\right) b_{\nu}^{*}\left(k_{2}{ }^{\prime}\right) b^{\nu}\left(k_{1}\right)\right\} .
\end{aligned}
$$

\section{iii. Vector Boson-Scalar Boson Scattering}

Let $k, p$ be the momenta of the incoming vector and scalar boson, respectively, and $k^{\prime}, p^{\prime}$ be their outgoing momenta. Again there are four contributions, $M_{s}, M_{t}$, $M_{u}$, and $M_{\text {direct. }}$. In the $s$ and $u$ channels a vector boson is exchanged and it turns out that the various propagators, $\left\langle T^{*} A_{\mu} A_{\nu}\right\rangle,\left\langle T^{*} A_{\mu} \Phi\right\rangle$, and $\left\langle T^{*} \Phi \Phi\right\rangle$, occur only in the combination $\left\langle T^{*} B_{\mu} B_{\nu}\right\rangle$. We obtain the expression

$$
\begin{array}{r}
M_{s}=i^{2}\left\{-2 e m_{1} b^{* \mu}\left(k^{\prime}\right)+i e q^{\mu} \phi^{*}\left(k^{\prime}\right)\right\} i\left(g_{\mu \nu}+m_{1}{ }^{-2} q_{\mu} q_{\nu}\right) \\
\times\left(s-m_{1}^{2}\right)^{-1}\left\{-2 e m_{1} b^{\nu}(k)-i e q^{\nu} \phi(k)\right\},
\end{array}
$$

where $q=k+p$ and $s=-q^{2}$, and a similar expression for $M_{u}$. In the $t$ channel a scalar boson is exchanged, and we find that

$$
\begin{aligned}
M_{t}=i^{2}\left\{-3 f m_{0}\right\} i\left(t-m_{0}\right)^{-1}\{ & -2 e m_{1} b_{\mu}^{*}\left(k^{\prime}\right) b^{\mu}(k) \\
& \left.+e m_{1}^{-1}\left(t-m_{0}\right) \phi^{*}\left(k^{\prime}\right) \phi(k)\right\},
\end{aligned}
$$

where $t=-\left(k-k^{\prime}\right)^{2}$. Finally, the contribution of the quartic vertices is given by

$$
\begin{aligned}
M_{\mathrm{direct}}=i\left\{-2 e^{2}\right. & {\left[b_{\mu}{ }^{*}\left(k^{\prime}\right)-i m_{1}{ }^{-1} k_{\mu}{ }^{\prime} \phi^{*}\left(k^{\prime}\right)\right] } \\
\times & {\left.\left[b^{\mu}(k)+i m_{1}^{-1} k^{\mu} \phi(k)\right]-f^{2} \phi^{*}\left(k^{\prime}\right) \phi(k)\right\} . }
\end{aligned}
$$

Again the four contributions sum to the invariant expression

$$
\begin{gathered}
M_{\text {total }}=-2 i m_{1}{ }^{2}\left\{2 e ^ { 2 } ( s - m _ { 1 } { } ^ { 2 } ) ^ { - 1 } \left[b_{\mu}{ }^{*}\left(k^{\prime}\right) b^{\mu}(k)\right.\right. \\
\left.+m_{1}^{-2} p_{\mu}^{\prime} b^{* \mu}\left(k^{\prime}\right) p_{\nu} b^{\nu}(k)\right] \\
+3 f^{2}\left(t-m_{0}^{2}\right)^{-1} b_{\mu}{ }^{*}\left(k^{\prime}\right) b^{\mu}(k) \\
+2 e^{2}\left(u-m_{1}^{2}\right)^{-1}\left[b_{\mu}{ }^{*}\left(k^{\prime}\right) b^{\mu}(k)\right. \\
\left.\left.+m_{1}{ }^{-2} p_{\mu} b^{* \mu}\left(k^{\prime}\right) p_{\nu}{ }^{\prime} b^{\nu}(k)\right]\right\} \\
\quad-2 i e^{2} b_{\mu}^{*}\left(k^{\prime}\right) b^{\mu}(k) .
\end{gathered}
$$

A similar matrix element may be written down for the process, vector pair $\leftrightarrow$ scalar pair, by making appropriate interchanges of incoming and outgoing momenta and wave functions. 


\section{iv. Scalar Boson-Scalar Boson Scattering}

This is the only simple process in which no invariance problems arise in lowest order: The vertices which are involved contain no vector boson factors. We find that

$$
\begin{aligned}
M_{\text {total }}= & M_{s}+M_{t}+M_{u}+M_{\text {direct }} \\
= & -9 i f^{2} m_{0}^{2}\left\{\left(s-m_{0}^{2}\right)^{-1}+\left(t-m_{0}^{2}\right)^{-1}\right. \\
& \left.\quad+\left(u-m_{0}^{2}\right)^{-1}+\left(3 m_{0}^{2}\right)^{-1}\right\} .
\end{aligned}
$$

\section{EQUIVALENT LAGRANGIAN}

In the previous section we have illustrated the Lorentz and gauge invariance of the model by the somewhat unsophisticated device of performing a few lowest order calculations. From a more sophisticated point of view we remark that Lorentz invariance may be proved by constructing the generators of the Lorentz group and verifying their commutation relations. Provided that the Lagrangian (1) is first properly symmetrized, the proof goes through as in quantum electrodynamics ${ }^{37}$; spontaneous breakdown of the internal symmetry is irrelevant to the argument, which depends only on the equal-time commutators of products of field operators.

Concerning gauge invariance we remark that our result, that (in lowest order at least) decay and scattering amplitudes depend only on the gauge-invariant vector wave functions $b_{\mu}(k, \sigma)$, suggests that it must be possible to rewrite the theory in a form in which only gauge-invariant variables appear. Indeed, if one were shown only the expressions (17)-(20), he would guess that they had been derived from an interaction Lagrangian given by

$$
\mathfrak{L}_{\text {int }}{ }^{\prime}=-e m_{1} B_{\mu} B^{\mu} \chi-\frac{1}{2} f m_{0} \chi^{3}-\frac{1}{2} e^{2} B_{\mu} B^{\mu} \chi^{2}-\frac{1}{8} f^{2} \chi^{4} .
$$

We shall now show that the expressions (7) and (21) are equivalent by finding a transformation of the total Lagrangian (1) which takes the one into the other.

We note that the gauge dependence of the classical dynamic variables may be expressed in the form

$$
\begin{aligned}
\Phi_{1}(x) & =R(x) \cos \Theta(x), \\
\Phi_{2}(x) & =R(x) \sin \Theta(x), \\
A_{\mu}(x) & =B_{\mu}(x)-e^{-1} \partial_{\mu} \Theta(x),
\end{aligned}
$$

where $R(x)$ and $B_{\mu}(x)$ are gauge invariant variables and the transformations (4) take the simple form, $\Theta(x) \rightarrow \Theta(x)-\Lambda(x)$. The classical Lagrangian (1), expressed in terms of the new variables, takes the form

$$
\begin{aligned}
\mathscr{L}^{\prime}=-\frac{1}{4} F_{\mu \nu} F^{\mu \nu}-\frac{1}{2} g^{\mu \nu} \partial_{\mu} R \partial_{\nu} R & \\
& -\frac{1}{2} e^{2} B_{\mu} B^{\mu} R^{2}+\frac{1}{4} m_{0}^{2} R^{2}-\frac{1}{8} f^{2} R^{4} .
\end{aligned}
$$

Gauge invariance here has ensured the disappearance

\footnotetext{
${ }^{37}$ See B. Zumino, J. Math. Phys. 1, 1 (1960).
}

of the variable $\Theta$ from the scene. [What we have done is to exploit the freedom which local $U(1)$ invariance gives us to "rotate" the entire two-component field $\Phi_{a}(x)$ onto one of the "axes." In a theory with only global $U(1)$ invariance this rotation cannot be performed on the entire field but only on the static solution (3).] The existence of the solution $B_{\mu}=0, R^{2}$ $=m_{0}^{2} / f^{2}$ suggests the substitution $R(x)=\eta+\chi(x)$. In this way, we find immediately that, apart from an additive constant, $\mathfrak{L}^{\prime}=\mathfrak{L}_{0}^{\prime}+\mathfrak{L}_{\text {int }}{ }^{\prime}$, where

$\mathfrak{L}_{0}^{\prime}=-\frac{1}{4} F_{\mu \nu} F^{\mu \nu}-\frac{1}{2} m_{1}^{2} B_{\mu} B^{\mu}-\frac{1}{2} g^{\mu \nu} \partial_{\mu} \chi \partial_{\nu} \chi-\frac{1}{2} m_{0}^{2} \chi^{2}$.

The expression (24) is the same as (6), except that the exactly gauge-invariant variables $B_{\mu}$ and $\chi$ which we have just defined replace their interaction picture counterparts.

We conjecture that the equivalence demonstrated here between the classical Lagrangians (1) and (23) may be extended to the corresponding quantum mechanical operators, provided that careful attention is given to the ordering problems which may arise, for example, in the definition of the current $j_{\mu}{ }^{38}$

\section{DISCUSSION}

The foregoing considerations illustrate our contention in Refs. 15 and 27 that the extension of a spontaneously broken internal symmetry of a Lorentz-invariant Lagrangian from global to local transformations not only may but actually does change zerons into the longitudinal states of massive vector bosons. Since we believe the value of this simple model to lie in the insight which it may give into this phenomenon when one looks at it as simple-mindedly as we have here, we shall not go into more difficult questions, such as radiative corrections and renormalization, in the present paper.

We note that in this model the original symmetry is almost unrecognizable in the physical states. Even without the gauge field coupling, the invalidation of the usual argument leading to the conservation of $Q(t)^{39}$ by the asymptotic behavior of the term $\left\langle\Phi_{2}\right\rangle \partial_{\mu} \Phi_{1}$ in $j_{\mu}$ destroys the commutativity of $Q$ with the Hamiltonian: Consequently, the one-particle states are not eigenstates of $Q$ and the masses within the $\Phi_{a}$ multiplet are not degenerate, but at least the multiplet structure remains. The gauge field coupling obscures even the multiplet structure: The scalar doublet is now incomplete, having lost its formerly massless member to form the longitudinal polarization of the vector singlet.

${ }^{38}$ J. Schwinger, Phys. Rev. Letters 3, 296 (1959); K. Johnson, Nucl. Phys. 25, 431 (1961).

${ }^{39}$ In passing, we remark that this feature of spontaneous breakdown theories seems to call into question the validity of the results obtained on the basis of chirality conservation by Nambu and his collaborators. See Y. Nambu and D. Lurié, Phys. Rev. 125, 1429 (1962); Y. Nambu and E. Shrauner, ibid. 128, 862 (1962); E. Shrauner, ibid. 131, 1847 (1963). 
In view of the rather drastic nature of the symmetry breakdown which we have just summarized, it is of interest to inquire what happens when this system is coupled to a second in a Lagrangian which retains local $U(1)$ invariance and contains no additional mechanisms for spontaneous breakdown. To be specific, let us take the second system to be a pair of "baryons" of "charges" $\pm \frac{1}{2}$, together with their antiparticles, and let us assume that the $\Phi$-baryon interaction is of the Yukawa type. The total Lagrangian is then given by

$$
\mathfrak{L}_{\text {total }}=\mathfrak{L}(A, \Phi)-\bar{\psi}_{a}\left(\gamma^{\mu} \nabla_{\mu}+M\right) \psi_{a}+g\left[\Phi_{1}\left(\bar{\psi}_{1} \psi_{2}+\bar{\psi}_{2} \psi_{1}\right)\right.
$$$$
\left.+\Phi_{2}\left(\bar{\psi}_{2} \psi_{2}-\bar{\psi}_{1} \psi_{1}\right)\right] \text {, }
$$

in which $\mathscr{L}(A, \Phi)$ is the expression (1), $\boldsymbol{\nabla}_{\mu} \psi_{1}=\partial_{\mu} \psi_{1}$ $-\frac{1}{2} e A_{\mu} \psi_{2}, \nabla_{\mu} \psi_{2}=\partial_{\mu} \psi_{2}+\frac{1}{2} e A_{\mu} \psi_{1}$ and we have, without loss of generality, made a choice of a phase angle in writing down the invariant Yukawa term. But for the presence of this last term, the Lagrangian would be invariant under global $U(1)$ transformations on $\Phi$ and $\psi$ independently; that is, the symmetry would be $U(1)$ $\times U(1)$ and the currents $j_{\mu}(\Phi)$ and $j_{\mu}(\psi)$ would be separately conserved. Thus, despite the nonconservation of $Q(\Phi)$ brought about by the structure of the first term in (25), there would still be conservation of $Q(\psi)$.

The Yukawa term reduces the symmetry to $U(1)$, the divergences of the individual currents now being given by

$$
\begin{aligned}
\partial_{\mu} j^{\mu}(\psi) & =g\left[\Phi_{1}\left(\bar{\psi}_{1} \psi_{1}-\bar{\psi}_{2} \psi_{2}\right)+\Phi_{2}\left(\bar{\psi}_{1} \psi_{2}+\bar{\psi}_{2} \psi_{1}\right)\right] \\
& =-\partial_{\mu} j^{\mu}(\Phi) .
\end{aligned}
$$

We observe that spontaneous breakdown of the symmetry in the $\Phi$ system breaks the symmetry of the $\psi$ system to an extent which depends on the coupling constant $g$. In the spirit of the perturbative approach which we have been using, we may evaluate the major part of the effects on the $\psi$ system by replacing $\Phi$ by its vacuum expectation value. Then in Eq. (25) the term $g \eta\left(\bar{\psi}_{2} \psi_{2}-\bar{\psi}_{1} \psi_{1}\right)$ removes the baryon mass degeneracy, and Eq. (26) becomes

$$
\partial_{\mu} j^{\mu}(\psi)=g \eta\left(\bar{\psi}_{1} \psi_{2}+\bar{\psi}_{2} \psi_{1}\right)+\text { higher order terms. }
$$

If we were to modify the Lagrangian (25) by adding to it such $U(1)$ invariant baryon-antibaryon interactions as would produce a doublet of low-mass scalar bound states with wave functions transforming as $\bar{\psi}_{1} \psi_{2}+\bar{\psi}_{2} \psi_{1}$ and $\bar{\psi}_{2} \psi_{2}-\bar{\psi}_{1} \psi_{1}$, then Eq. (27) would be approximately a partial conservation law of the type which has proved so successful for the axial currents of the weak interactions..$^{40}$ Moreover, the current $j_{\mu}(\psi)$ interacts with itself via the massive intermediate vector boson which the $\Phi-A_{\mu}$ coupling produces.

There appears to be some hope that the basic ingredients of our model, namely the combination of spontaneous symmetry breakdown with the gauge principle, may provide the basis for an understanding of the broken symmetries of high-energy physics. In a subsequent paper we shall discuss models in which the breakdown of higher symmetries such as $S U(3)$ is treated in the same fashion.

\section{ACKNOWLEDGMENTS}

I have learned much about the Goldstone theorem from discussions with Dr. G. S. Guralnik, Dr. C. R. Hagen, Dr. T. W. B. Kibble, and Dr. R. F. Streater. I wish to thank Professor Bryce and Professor Cécile DeWitt for the hospitality of the Institute of Field Physics at the University of North Carolina, where this work was completed.

${ }^{40}$ It would be the type of partial conservation law proposed by Y. Nambu, Phys. Rev. Letters 4, 380 (1960) and by M. Gell-Mann, Phys. Rev. 125, 1067 (1962), rather than that proposed by M. Gell-Mann and M. Lévy, Nuovo Cimento 16, 705 (1960), in which the low-lying scalar or pseudoscalar states are treated as elementary rather than composite in the context of a field theory. 